\title{
Quantisation of the Multidimensional Rotor
}

\author{
E. Abdalla $a^{a}$ and R. Banerjee ${ }^{b}$ \\ ${ }^{a}$ Instituto de Física-USP, C.P. 66318, 05315-970 São Paulo, Brazil \\ ${ }^{b}$ S.N. Bose National Centre for Basic Sciences \\ Block JD, Sector III, Salt Lake City, Calcutta 700.091 India
}

Received on 9 September, 2000

\begin{abstract}
We reconsider the problem of quantising a particle on the $D$-dimensional sphere. Adopting a Lagrangian method of reducing the degrees of freedom, the quantum Hamiltonian is found to be the usual Schrödinger operator without any curvature term. The equivalence with the Dirac Hamiltonian approach is demonstrated, either in the cartesian or in the curvilinear basis. We also briefly comment on the path integral approach.
\end{abstract}

The problem of the quantisation of the rotor has been studied since decades, but remains a subject of intense debate. De Witt[1] studied the path integral quantization, and proposed that a term proportional to the curvature should be included in the Hamiltonian. There are, however, problems connected to the definition of the path integral using curvilinear coordinates, since quantising in curvilinear coordinates leads to new terms, even for free particles. Edwards and Gulyaev[2] carefully considered that problem, and showed that due to problems intrinsically connected to the path integral formulation[3], path integration quantisation may lead to different results.

The Dirac formulation[4] has also been undertaken[5]. In this case one faces the question of operator ordering, a question which can only be tackled using definite prescriptions based on general arguments as hermiticity and general coordinate invariance. The Laplace-Beltrami operator has been obtained without curvature terms in e.g. [5], for the special case of a three-dimensional rotor.

Besides the early developments[2], there are special definitions of the path integral on the surface of the sphere which do not give rise to the curvature term[6]. Several diferent results have been obtained in the literature[6, $7,8,9,10]$.

In spite of all these developments, the status of the problem is very confusing, and there have been many papers claiming a rejection of the Dirac formalism[6] an intrinsic difference between path integral formulation and operator formalism[7], or advocating different quantization schemes[9]. The importance of the question can be appreciated from the fact that it has implications in curved space quantization, when defining the Wheeler-de-Witt equation[11], and in the quantisation of sigma model Lagrangians.

The aim of the present paper is to analyse the prob- lem directly in terms of reduced coordinates by solving the constraint, bypassing the ambiguities inherent in either the Dirac or path integral approaches. The classical reduced space is therefore obtained in a straightforward manner. We then pass to the quantum formulation by using the Laplace-Beltrami construction, leading to the usual Schrödinger operator without any curvature term. The connection of our results with the Dirac approach is then established. In particular, the role of the Maskawa-Nakajima[12] theorem in the Dirac formalism in the cartesian basis is elaborated. We conclude mentioning the path integral formulation, where the counterpart of the above approach is the special time slicing of the integral as explained by T. D. Lee[13].

The Lagrangian for a particle of unit mass constrained to move on the surface of a $D$-dimensional sphere of radius $R$ is given by

$$
\mathcal{L}=\frac{1}{2} \dot{x}_{\alpha} \dot{x}_{\alpha}-\lambda\left(x_{\alpha} x_{\alpha}-R^{2}\right) \quad \alpha=1 \cdots D,
$$

where the constraint is implemented by a Lagrange multiplier. Thus we use Dirac's constraint analysis. Apart from the fact that Dirac brackets are plagued by ordering ambiguities, the extraction of the physical variables of the system is not very transparent $[5,6,7,10]$.

Here we shall adopt an alternative canonical approach developed by one of us[14] which is based on a Lagrangian reduction by systemmatically eliminating the unphysical variables using the constraint. Eliminating $x_{D}$ we obtain

$$
\mathcal{L}_{r}=\frac{1}{2} g_{i j} \dot{x}_{i} \dot{x}_{j}=\frac{1}{2}\left(\delta_{i j}+\frac{x_{i} x_{j}}{R^{2}-\vec{x}^{2}}\right) \dot{x}_{i} \dot{x}_{j} .
$$

The conventional canonical formalism is now applicable, and the canonical Hamiltonian is

$$
\mathcal{H}=p_{i} \dot{x}_{i}-\mathcal{L}_{r}=\frac{1}{2} p_{i} g^{i j} p_{j}
$$


which gives the final expression for the classical reduced Hamiltonian in terms of the momenta conjugate to $x_{i}$.

In order to perform the quantisation the above Hamiltonian is replaced by the corresponding LaplaceBeltrami operator, being defined as

$$
\hat{\mathcal{H}}=\mathcal{O}_{L B}=\frac{1}{2} g^{-1 / 4} \hat{\pi}_{i} g^{1 / 2} g^{i j} \hat{\pi}_{j} g^{-1 / 4},
$$

where $\hat{\pi}$ is the quantum momentum operator

$$
\hat{\pi}_{i}=-i \hbar g^{-1 / 4} \vec{\partial} g^{1 / 4},
$$

and $g$ is the determinant of the metric $g \equiv \operatorname{det} g_{i j}=$ $\frac{R^{2}}{R^{2}-\vec{x}^{2}}$. This transition from classical to quantum theory is based on hermiticity, general coordinate invariance and preservation of the symmetry properties.

It is now simple to obtain the quantum Hamiltonian,

$$
\hat{\mathcal{H}}=-\frac{1}{2} \sqrt{R^{2}-\vec{x}^{2}} \vec{\partial}_{i}\left(\delta_{i j}-\frac{x_{i} x_{j}}{R^{2}}\right)\left(R^{2}-\vec{x}^{2}\right)^{-1 / 2} \vec{\partial}_{j} \equiv \sum_{\alpha \beta} \frac{L_{\alpha \beta}^{2}}{2 R^{2}}
$$

where the angular momentum operator is given by

$$
\begin{aligned}
L_{i j} & =x_{i} p_{j}-x_{j} p_{i}=-i \hbar\left(x_{i} \partial_{j}-x_{j} \partial_{i}\right), \\
L_{i D} & =-\sqrt{R^{2}-\vec{x}^{2}} p_{i}=-L_{D i}=i \hbar \sqrt{R^{2}-\vec{x}^{2}} \partial_{i}
\end{aligned}
$$

The conjugate momentum $p_{D}$ does not exist in the reduced variables. Thus the quantum Hamiltonian is the conventional Schrödinger operator without any curvature term. This is our central result.

The analysis was carried out in the cartesian basis, but it is instructive to repeat it in the curvilinear basis, illuminating the connection with the conventional Dirac approach. Using the standard curvilinear coordinates the original Lagrangian is given by

$$
\mathcal{L}=\frac{1}{2}\left\{\dot{r}^{2}+r^{2} \dot{\varphi}_{1}^{2}+\cdots r^{2} \dot{\varphi}_{D-1}^{2} \sin ^{2} \varphi_{1}^{2} \cdots \sin ^{2} \varphi_{D-2}\right\}-\lambda(r-R) .
$$

In constrast to the cartesian analysis, the solution of the constraint (a constant radius) here is trivial and the reduced Hamiltonian obtained by following the previous steps is worked out to be

$$
\mathcal{H}=\frac{1}{2} \sum g^{a b} \pi_{a} \pi_{b}
$$

with the inverse diagonal metric $g_{a b} \equiv g^{-1^{a b}}$ given by

$$
g_{a b}=R^{2}\left(1, \sin ^{2} \varphi_{1}, \sin ^{2} \varphi_{1} \sin ^{2} \varphi_{2}, \cdots, \sin ^{2} \varphi_{1} \sin ^{2} \varphi_{2} \cdots \sin ^{2} \varphi_{n-2}\right) .
$$

The quantum Hamiltonian, which is given by the corresponding Laplace-Beltrami operator, reduces to

$$
\hat{\mathcal{H}}=-\frac{\hbar^{2}}{2 R^{2}} \sum_{i=1}^{n-1} \prod_{j=1}^{i-1} \frac{1}{\sin ^{2} \varphi_{j}} \frac{1}{\left(\sin \varphi_{i}\right)^{n-i-1}} \frac{\partial}{\partial \varphi_{i}}\left(\sin \varphi_{i}\right)^{n-i-1} \frac{\partial}{\partial \varphi_{i}}
$$

which corresponds to (6). To compare with the Dirac formalism we start from the constrained Lagrangian (9). The set of primary constraints is now given by

$$
\Omega=\pi_{\lambda}=0 \quad, \quad \Omega_{1}=r-R \approx 0 \quad, \quad \Omega_{2}=\pi_{r} \approx 0 .
$$


The unphysical canonical set $\left(\lambda, \pi_{\lambda}\right)$ associated with the Lagrange multiplier is ignored. This leaves us with a pair of second class constraints, $\Omega_{1}$ and $\Omega_{2}$, forming a canonical set,

$$
\left\{\Omega_{i}, \Omega_{j}\right\}=\epsilon_{i j}
$$

The special form of the constraints allows a straightforward application of the Maskawa-Nakajima theorem[12] to extract the physical variables and the Hamiltonian without the need of any explicit computation of Dirac brackets. Using this theorem, it is simple to show that the canonical pairs are given by $\varphi_{i}, \pi_{\varphi_{i}}$. In other words, the Dirac brackets among these variables is equal to their Poisson brackets. The physical Hamiltonian is now obtained from the canonical Hamiltonian by passing on to the constraint shell. This is found to coincide with the reduced Hamiltonian (10). The quantum Hamiltonian is then reobtained from the corresponding Laplace-Beltrami operator.

The Dirac analysis of this problem in the cartesian basis is quite nontrivial, since the constraint algebra is no longer canonical. The Maskawa-Nakajima theorem allows finding the canonical transformation which enables the extraction of the canonical pair of variables without any further ambiguities. The constraint structure is given by

$$
\Omega_{1}=x_{i} x_{i}-R^{2}=0 \quad \Omega_{2}=x_{i} p_{i}=0
$$

The Maskawa Nakajima canonical tr. is just the transformation from the cartesian to the spherical basis. In these redefined variables the physical hamiltonian is given by,

$$
\mathcal{H}_{\text {phys }}=\frac{1}{2 R^{2}}\left(\pi_{\theta}^{2}+\frac{1}{\sin ^{2} \theta} \pi_{\varphi}^{2}\right)
$$

This completes the classical reduction. Since the above Hamiltonian is expressed in terms of canonical pairs, the Laplace-Beltrami construction goes through and we exactly reproduce the canonical Hamiltonian.

The main conclusion of the present work is that the quantum Hamiltonian for the multidimensional rotor is given by the pure Schrödinger operator without any curvature term. This result was obtained in the Lagrangian formalism [16] by directly solving the constraint and reducing the unwanted degrees of freedom. The usual ambiguities of the Dirac approach in the cartesian basis were avoided by taking recourse to the powerful Maskawa-Nakajima theorem [12].

It is essential to point out that the transition from the classical to the quantum theory was done by exploting the Laplace-Beltrami construction, enforcing the conditions of hermiticity, general coordinate invariance, and preservation of the classical symmetries. While the passage from the classical to the quantum theory is riddled with ambiguities, the Laplace-Beltrami operator provides a desirable quantum hamiltonian. Curvature terms are ruled out since these are incompatible with the third condition. In particular, they violate the conservation of the generator of the equilong transformation group [9]. Yet another way of seeing the importance of the Laplace-Beltrami construction is to compare it with the path integral formalism [13]. The specific time slicing prescription used in [13] is known to yield consistent results for the path integral either in the cartesian or curvilinear basis. Using the LaplaceBeltrami operator in the canonical quantisation of nonabelian gauge theories [15] naturally led to the interaction terms originally postulated by Schwinger [16] and subsequently derived in the path integral framework [13]. The different methods of reducing the unwanted degrees of freedom in the lagrangian and hamiltonian approaches, yield completely consistent results. This is the important finding of the present work which serves to dispel at least some of the confusion and controversy existing in the literature.

A possible approach of discussing systems with second class constraints is to embed them into a first class system. Details of this procedure in the present context have been given in [17] where the invariance of the quantum theory under reparametrisation of the constraint surface has also been studied. Regarding the path integral formulation, the basic problem here stems from the fact that the definition of the path integral in curvilinear coordinates is rather tricky and subtle. An apparent clash between the canonical and (a naive) path integral formulation is already seen in the simplest of examples, namely a free non relativistic particle in two dimensions[18].

Thus, a clear computation as the one performed by Kleinert[6] leads to the correct result. We however point out that a distinction can be made, so that it is possible to treat the particle either on or near the surface of the sphere. For the former case the boundary term disappears[6] but in the latter, such a term exists. Since in the present analysis the constraints are always strongly enforced, we are confined to the case of the particle exactly on the sphere. A recent calculation from a more mathematical point of view also confirms our result[19].

Acknowledgements: this work has been partially supported by Conselho Nacional de Desenvolvimento Científico e Tecnológico and Fundação de Amparo a Pesquisa do Estado de São Paulo, Brazil. E.A. wishes to thank the S.N. Bose National Centre for Basic Sciences, Calcutta, India, for the hospitality.

\section{References}

[1] B. De Witt Phys. Rev. 85 (1952) 653; Rev. Mod. Phys. 29 (1957) 377.

[2] S. F. Edwards and Y. V. Gulyaev Proc. Roy. Soc. A279 (1964) 229 
[3] R. J. Rivers Path integral methods in Quantum Field Theory, Cambridge monographs in Mathematical Physics, 1987.

[4] P. A. M. Dirac Lectures on Quantum Mechanics New York Belfer Graduate School of Sciences, Yeshiva University.

[5] N. K. Falck and A. C. Hirshfeld Eur. J. Phys. 4 (1983) 5.

[6] H. Kleinert Path Integrals in Quantum mechanics Statistical and Polymer Physics, World Scientific 1995; H. Kleinert and S. V. Shabanov Phys. Lett. A232 (1997) 327.

[7] A. Foerster, H. O. Girotti abd P. S. Kuhn Phys. Lett. A 195 (1994) 301.

[8] M. S. Marinov Phys. Rep. 60 (1980) 1.

[9] M. Omote and H. Sato Prog. Theor. Phys. 47 (1972) 1367.
[10] A. Saa Class. Quantum Grav. 14 (1997) 385.

[11] O. Bertolami Phys. Lett. A154 (1991) 225.

[12] T. Maskawa and H. Nakajima Prog. Theor. Phys. 56 (1976) 1295.

[13] T. D. Lee Particle Physics and Introduction to Field Theory Harwood Academic Publishers, 1990.

[14] R. Banerjee hep-th/9607199.

[15] N.K. Falck and A.C. Hirshfeld Ann Phys (NY) 144 (1982) 34

[16] J. Schwinger Phys Rev 125 (1962) 1043

[17] J.R. Klauder and S.V. Shabanov Nucl Phys B511 (1998) 713

[18] A. K. Kapoor Phys. Rev. D29 (1984) 2339.

[19] H. Grundling and C. A. Hurst hep-th/9712052 